Objective.-The aim of this study is to determine whether there is a difference in the cause - acute or overuse - and severity of injuries evaluated by a physician compared with injuries that are not. We also intend to describe the prevalence, type, and anatomical location of injuries sustained by rock climbers.

Methods.-A retrospective, cross-sectional, anonymous survey was distributed at popular outdoor climbing locations in North America. Descriptive statistics and $\chi^{2}$ tests were used to describe the study sample and identify factors associated with medical treatment.

Results.-Of 192 people, $70.8 \%$ reported at least 1 injury in the previous 2 years. Injuries from acute events $(69.8 \%)$ were more common than those from overuse $(35.3 \%)$. Strenuous moves caused most acute injuries (69.5\%), followed by falls (27.4\%). The most commonly injured body part was the finger (38.2\%), followed by the shoulder $(15.4 \%)$. A physician evaluated $41.9 \%$ of all injuries. Although the injuries evaluated by a physician were more severe $(P<.0001)$, there was no significant difference in the cause of the injury, either acute or overuse.

Conclusions.-The findings suggest injury severity, rather than cause, appears to be the main determinant in the decision to seek professional care. Although acute trauma is the more common cause of injuries, a large proportion of all climbingrelated injuries are associated with overuse. As overuse and chronic injuries are often overlooked in the literature, our findings that many overuse injuries are serious enough to require medical attention suggest a need for further inquiry into the risk factors and behaviors associated with these injuries.

Nathan Furst-Nichols, BS Courtney Jones, MPH, PhD

Erik Rueckmann, MD

Mark Mirabelli, MD

Rochester, NY, USA

\section{Irukandji-Like Syndrome in the Caribbean Sea, Puerto Rico: Case Report}

Objective.-Irukandji syndrome has been described as a painful envenoming event caused by different species of jellyfish, mainly in the Cubozoa class. This hypercatecholaminergic condition, with recorded fatalities and severe systemic symptoms, has been described mostly on Australian seas. To our knowledge, Irukandji syndrome has not been reported in Puerto Rico or the Caribbean Sea. Our report describes 2 cases of marine envenomation causing this syndrome in Puerto Rican divers. The aim of this case report is to raise awareness of this syndrome in the Caribbean area, thus preventing misdiagnosis, underreporting, and improper treatment strategies.

Case report.-A 40-year-old man was diving east of Puerto Rico island. After the ascend, he was struck by a box-shaped jellyfish, clear to light blue in color, with long tentacles. The diver then had lower limb weakness, severe back pain, fast heart rate, palpitations, agitation, memory impairment, disorientation, nausea, shortness of breath, diaphoresis, involuntary tremors, and an intense feeling of doom. Hyperbaric treatment was given but was unsuccessful. The Poison Control Center of Puerto Rico made the diagnosis of Irukandji-like syndrome.

Conclusion.- The Irunkandji syndrome can be a lifethreatening condition if it is treated incorrectly or is misdiagnosed, as could happen if it occurs outside the usual areas. In the Caribbean area, we should be more aware of this syndrome and include it in the differential diagnosis when a patient comes to us with hypercholinergic symptomatology.

Isabel Algaze, MD

Joanna Mercado, MD

Pedro Arroyo, MD

Ponce, Puerto Rico

\section{Utilization of Wrist Protection by Snowboarders}

Introduction.-Snowboarding injuries account for nearly 70,000 emergency department visits annually. Wrist injuries are the most common injury sustained while snowboarding, representing $20 \%$ to $40 \%$ of all injuries. Despite a growing body of evidence demonstrating that wearing wrist protection can reduce this risk by as much as half, the use of protective equipment remains low.

Objective.-The purpose of this study is to determine the utilization of wrist protection by snowboarders and to examine underlying attitudes toward use of protective gear.

Methods.-This is a cross-sectional study of data collected from anonymous, voluntary responses to questionnaires distributed to snowboarders visiting 1 of 3 designated study sites on the East Coast during the 2013-2014 winter season. Snowboarders under the age of 13 years were excluded from the study.

Results.-Of 245 snowboarders approached, 230 (93.8\%) agreed to participate. Participants ranged in age from 13 to 58 years, with a median age of 22 years; $77.2 \%$ of respondents were male and $22.8 \%$ were female. Snowboarding experience was self-selected as novice $(7.9 \%)$, beginner $(25.8 \%)$, intermediate $(31.9 \%)$, or expert $(34.5 \%)$. Overall, $74.1 \%$ of participants reported never wearing wrist protection, and only $9.8 \%$ indicated that they always wear wrist protection. The top 2 reasons selected for not using wrist protection were, "I don't see the need" (48.1\%) and "I didn't know wrist protectors were available for snowboarding" (37.3\%). Of note, $54.2 \%$ of respondents stated that they would wear wrist protectors if available. Sex $(27.55 \%$ male vs $70.37 \%$ female, $P<.0002)$ and helmet use $(30.30 \%$ helmeted vs $13.56 \%$ unhelmeted, $P<$ $.01)$ were found to be significant predictors of willingness to use wrist protectors if available.

Conclusions.-Although wearing wrist protectors has the potential to reduce risk and severity of wrist injury while snowboarding, utilization rates remain low. This study has identified some prevailing attitudes in this population that can be used as targets of behavior change.

R. Alissa Mussell, MD Hollynn L. Larrabee, MD 